an older age, earlier age at initiation, previous quit attempts) or psychological factors (e.g. stress/anxiety, degree of nicotine dependence) [8, 9]. Therefore, besides evaluating a patient's likelihood of smoking resumption, active screening for smoking, both when listed for LTx and during post-LTx follow-up, should be performed. For the patients resuming smoking, a standardised smoking cessation plan should be implemented. Patients' relatives, who most often continue smoking after LTx, must be recommended to stop smoking. At present, however, most LTx centres neither monitor smoking nor perform postLTx smoking cessation counselling.

The risk of smoking for worsening post-LTx outcome (i.e. by bronchiolitis obliterans syndrome, cancer or cardiovascular mortality) is currently unclear, yet long-term survival in heart transplantation is impaired by smoking due to chronic allograft rejection (graft coronary artery disease) [6]. Therefore, this ought to be investigated after LTx through a prospective study with repeated screening for smoking. The gold standard for screening, however, is currently unknown. Although a questionnaire is subjective; it has a good sensitivity/specificity to detect smoking and a good correlation with the COT test [10], a more objective assessment of the patient's nicotine-exposure [4, 11]. Despite being sensitive, COT conversely has a lower specificity as it may be falsely positive because of NRT or intensive second-hand smoking. False-negative values after pausing smoking are also possible as the half-life of COT is about $16 \mathrm{~h}$ [11]. False-negative tests could be prevented by repeated or unexpected COT tests. Although a lot less sensitive, eCO may be adequate to confirm the questionnaire or COT findings, but probably not to discriminate current from secondhand or nonsmokers as eCO may also be increased due to elevated airway neutrophilia after LTx [4].

In conclusion, the prevalence of post-LTx smoking is higher than generally assumed and active screening, both pre- and post-LTx, is crucial to detect smoking resumption. A standardised questionnaire and (preferably repeated) testing of COT is probably the best screening method. To confirm these findings we recommend eCO monitoring. Implementing a standardised smoking cessation plan after LTx must be considered. Finally, the importance of smoking resumption for the outcome after LTx should be investigated in a well-designed prospective study.

R. Vos* , K. De Vusser ${ }^{*}$, , V. Schaevers*, , A. Schoonis*, V. Lemaigre*, F. Dobbels*, K. Desmet", B.M. Vanaudenaerde*, D.E. Van Raemdonck*, L.J. Dupont* and G.M. Verleden*
*Lung Transplantation Unit, and "Laboratory Medicine, Katholieke Universiteit Leuven and University Hospital Gasthuisberg, Leuven, Belgium, "These authors contributed equally to the manuscript.

Correspondence: G.M. Verleden, Lung Transplantation Unit, University Hospital Gasthuisberg, 49 Herestraat, B-3000 Leuven, Belgium. E-mail: geert.verleden@uz.kuleuven.ac.be

Support Statement: This study is supported by the Research Foundation-Flanders (FWO G.0518.06, G.0643.08).

Statement of Interest: A statement of interest for G.M. Verleden can be found at www.erj.ersjournals.com $/ \mathrm{misc} /$ statements.dtl

\section{REFERENCES}

1 Verleden GM, Dupont LJ, Van Raemdonck DE, et al. Lung transplantation: a 15-year single-center experience. Clin Transpl 2007; 121-130.

2 Van der Heide F, Dijkstra G, Porte RJ, et al. Smoking behavior in liver transplant recipients. Liver Transpl 2009; 15: 648-655.

3 Zieliska-Danch W, Wardas W, Sobczak A, et al. Estimation of urinary cotinine cut-off points distinguishing non-smokers, passive and active smokers. Biomarkers 2007; 12: 484-496.

4 Vos R, Cordemans C, Vanaudenaerde BM, et al. Exhaled carbon monoxide as a noninvasive marker of airway neutrophilia after lung transplantation. Transplantation 2009; 87: 1579-1783.

5 Leete R, Fox AJ. Registrar General's social classes: origins and uses. Popul Trends. 1977; 8: 1-7.

6 Botha $\mathrm{P}$, Peaston $\mathrm{R}$, White $\mathrm{K}$, et al. Smoking after cardiac transplantation. Am J Transplant 2008; 8: 866-871.

7 Nguyen PT, Galanti L, Pirson Y, et al. Identification of current smokers among renal transplant recipients. Nephrol Dial Transplant 2007; 22: 1974-1978.

8 Caponnetto P, Polosa R. Common predictors of smoking cessation in clinical practice. Respir Med 2008; 102: 1182-1192.

9 Dobbels F, Verleden G, Dupont L, et al. To transplant or not? The importance of psychosocial and behavioural factors before lung transplantation. Chron Respir Dis 2006; 3: 39-47.

10 Molina AJ, Fernández D, Delgado $M$, et al. Sensitivity and specificity of a self-administered questionnaire of tobacco use; including the Fagerström test. Int J Nurs Stud 2010; 47: 181-189.

11 Benowitz NL. Clinical pharmacology of nicotine: implications for understanding, preventing, and treating tobacco addiction. Clin Pharmacol Ther 2008; 83: 531-541.

\title{
New DNAH11 mutations in primary ciliary dyskinesia with normal axonemal ultrastructure
}

\section{To the Editors:}

In primary ciliary dyskinesia (PCD; Mendelian Inheritance in Man database \#242650), a rare genetic disorder, the dysfunctional motility of cilia and impaired mucociliary clearance result in a myriad of clinical manifestations including recurrent infections of the respiratory tract, eventually causing lung damage, such as bronchiectasis, laterality defects and male infertility 
[1]. The heterogeneous clinical presentation of PCD and the limitations of transmission electron microscopy (TEM) to assess ultrastructural defects within the cilium may delay the diagnosis [2]. Ciliary beat frequency and pattern analyses and nasal nitric oxide (nNO) measurements are helpful [1, 3, 4], but only ciliogenesis in cultured cells has been reported as decisive for the diagnosis of the atypical PCD phenotype with normal axonemal ultrastructure $[1,5]$. Under these conditions, the identification of disease-causing mutations could overcome the current diagnostic limitations of TEM and improve our understanding of the biology and function of the cilium $[2,6$, 7]. For these reasons, we have assessed whether an analysis of the DNAH11 gene, in which some nonsense mutations have been reported to be associated with a normal axonemal ultrastructure but with an abnormal nonflexible beating pattern, reduced cilium bending capacity and a hyperkinetic beat [7], could be used to identify new mutations in three atypical PCD patients and thus be used in the diagnostic workup of these most difficult cases.

We observed three patients (A and B, sister and brother; and C, male), at the ages of 15 yrs and 5 months, 9 yrs and 4 months, and $8 \mathrm{yrs}$, respectively. The two siblings presented situs inversus. Both patients had chronic respiratory symptoms which required repeated treatment with antibiotics. Patient A also had neonatal purulent rhinitis with frequent relapses, chronic rhinosinusitis and otitis that required adenoidectomy and transtympanic drainage at the age of 6 yrs. Patient B also presented recurrent wheezy bronchitis and pneumonia. Patient $C$ had neonatal respiratory distress unrelated to any evident cause, situs inversus, partial inter-atrial defect, recurrent otitis and pneumonia from the age of 18 months.

Moreover, patient A presented bronchiectasis and atelectasis of the left middle lobe associated with fibrotic areas at chest highresolution computed tomography and pan-sinusitis at computed tomography scan. Pan-sinusitis and bronchioloectasis of the left middle lobe associated with fibrotic areas were also found in patient B, while a fibrotic area in the left middle lobe, maxillary and sphenoid sinusitis with agenesis of frontal sinuses were found in patient $C$.

In all three patients TEM evaluation showed few nonspecific abnormalities such as swollen cilia (7.7-13.6\%) and compound cilia $(0.8-1.5 \%)$, which are compatible with mild inflammation. None of the patients showed alterations of the central pair and dynein arm deficiencies [5-7]. Ciliary motion analysis showed an abnormal nonflexible beating pattern with a reduced cilium bending capacity in $49.9-76.5 \%$ of microscopic fields and a hyperkinetic beat of cilia up to $16 \mathrm{~Hz}$ (mean \pm SD normal values in our laboratory: $12.1 \pm 1.8 \mathrm{~Hz}$ ) with immotile cilia in $4-32 \%$ of microscopic fields, whereas a very low beat frequency resulted in the remaining fields. In all three patients functional ciliary evaluation after ciliogenesis in culture allowed us make a final diagnosis of PCD in relation to complete absence of either migration or rotation of spheroids [5]. $\mathrm{nNO}$ was low in all three patients (33.7, 26.9 and $19.8 \mathrm{ppb})$. Therefore, the diagnosis of PCD in these children relied on clinical features together with typical ciliary beat frequency and pattern phenotypes [1,3] and on ciliary functional analysis after ciliogenesis in culture [5], as well as on the low level of $\mathrm{nNO}[4,8]$.
Moreover, in all three patients, we identified novel DNAH11 compound heterozygous mutations, which were not found in the control population of 200 chromosomes. In particular, the two siblings carried the same compound heterozygous mutations in the DNAH11 gene (fig. 1a): a splice-site mutation (c.883-1G $>$ A) located in the acceptor splice site of exon 5 and a nonsense mutation (c.4145G >A, p.W1382X) located in exon 23. An analysis of parental samples revealed that the unaffected mother had transmitted the c.883-1G $>$ A mutation while the unaffected father had transmitted the p.W1382X mutation.

The third patient carried compound heterozygous mutations in the DNAH11 gene (fig. 1b): a mis-sense mutation (c.8135A $>\mathrm{G}$, p.H2712R) located in exon 50 and a mis-sense mutation (c.10284G $>$ A, p.G3429R) located in exon 64. An analysis of parental samples revealed that the unaffected mother had transmitted the p.H2712R mutation while the unaffected father had transmitted the p.G3429R mutation.

Obviously, genetic analysis represents a particularly important diagnostic instrument in atypical cases. A homozygous nonsense mutation in dynein heavy chain DNAH11 has previously been associated with immotile cilia despite normal axonemal structure in a subject with situs inversus [9]. Due to the concomitant presence of cystic fibrosis in this patient, some doubts were raised concerning the involvement of DNAH11 in this atypical case of PCD. However, DNAH11 mutations were subsequently confirmed in a German family that includes five individuals with PCD, and another with Kartagener syndrome, but with a normal ultrastructure of the respiratory cilia [7]. All these six individuals harboured compound heterozygous mutations in DNAH11.

Our results provide additional evidence of the possible role of new mutations in three patients with atypical PCD; and we can confirm that involvement of DNAH11, first observed in an inversus viscerum mouse model [10], is important for organ lateralisation since all our patients presented situs inversus.

Dynein heavy chains consist of an N-terminal stem which interacts with other dynein components and the head or motor domain. The motor contains six tandemly-linked AAA domains in the head, which form a ring. A stalk-like structure protrudes between AAA4 and AAA5 and terminates in a microtubulebinding site [11].

The splice acceptor site c.883-1G $>$ A mutation found in the first family occurs in intron 4 . In order to predict whether the splice mutations will lead to an abnormal splicing, we performed in silico analysis using Splice Site Prediction by Neural Network [12]. The analysis showed that the mutation may cause exon skipping, activation of different potential cryptic splice-sites and integration of part of intron 4 within the mRNA. The predicted aberrant mRNA could result in a frameshift and a premature stop codon which may lead to the synthesis of a truncated protein or to nonsense-mediated mRNA decay. To test how effectively this mutation affects the splicing, we should have performed cDNA analysis, but the respiratory epithelial cells obtained by nasal brush biopsies to isolate the RNA were not available.

The nonsense mutation p.W1382X located in exon 23 affects the N-terminal stem (1-1861 amino acids) of the protein. 
a) a a c t t a g c t t c a g

\begin{tabular}{cccc}
$*$ & L & $S$ & $F$ \\
\hline A A C T TARC TTCA G
\end{tabular}

A A C T TARCTTCA G
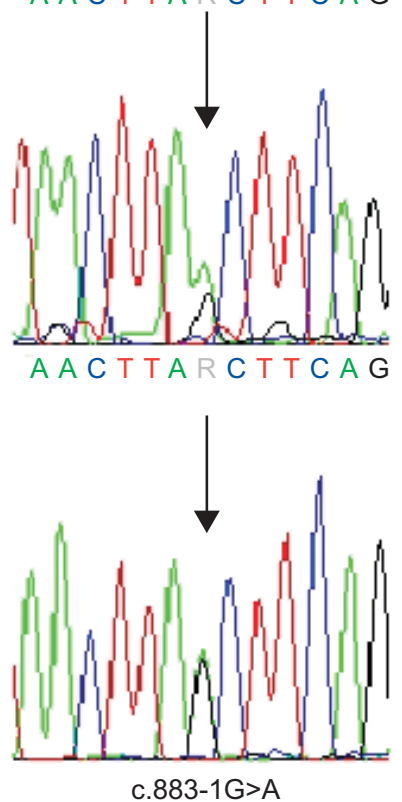

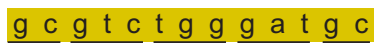

$\frac{R}{\text { GCG TCTRG WATGC }}$
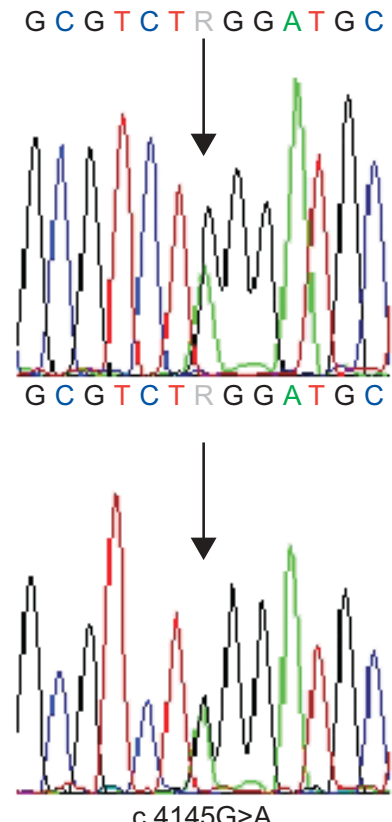

b) a a t t c c a c t a c a t

$\begin{array}{lllll}* & \text { I } & P & \mathrm{~L} & \mathrm{H}\end{array}$

A AT T C CRCTACAT

A A T T C C R C TA C AT
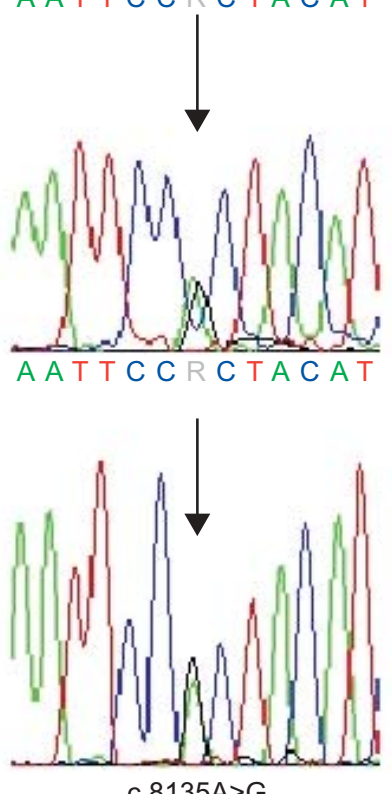

t a c g t c g g a c c c t

$\frac{Y \quad V \quad G \quad P}{\text { TACGTCRGACC C T }}$

TACGTCRGACCCT
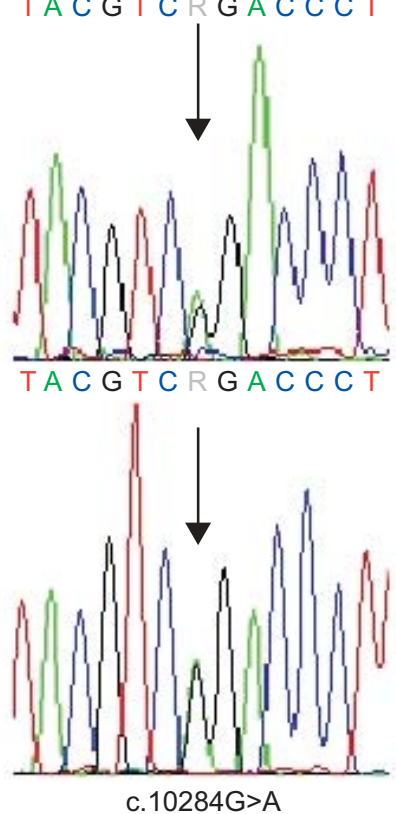

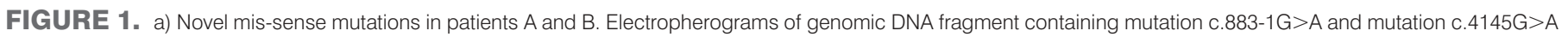

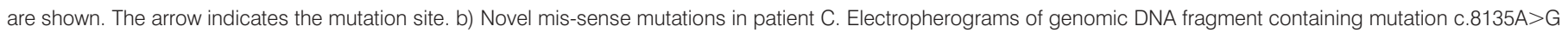

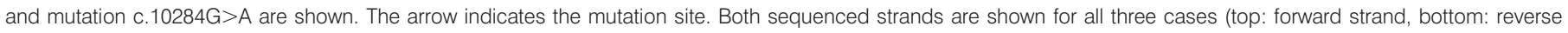
strand).

Therefore, the two mutations reported should truncate the entire C-terminal domain of the DNAH11 protein and if degraded by the nonsense-mediated mRNA decay pathway, they should lead to downregulate gene expression after the DNAH11 gene has been transcribed.

The mutations found in the second family are both mis-sense mutations and affect two different domains of the protein. The p.H2712R mutation affects the AAA3 domain (2479-2726 aa), while the p.G3429R mutation affects the region between the stalk-like structure (3079-3410 aa) and the AAA5 domain (3466-3693 aа).

In silico, we did an analysis using the PolyPhen program [13] to predict whether a nonsynonymous single-nucleotide substitution is expected to have a deleterious effect. PolyPhen considered it likely that both p.H2712R and p.G3429R would be damaging (PSIC score difference 3.352 and 2.739, respectively), suggesting a deleterious effect on protein conformation and function. These new variants can be categorised as pathogenic mutations and their severity is indicated by the severe phenotype of PCD in these patients.

All of the amino acid substitutions fall into highly conserved regions among different species and are not reported in the HGMD professional database [14].

The identification of the mutation in parents provides an opportunity for genetic counselling; and carrier testing for atrisk family members is an important tool for already-born children as well as for prenatal testing in the case of successive pregnancies.

In conclusion, our newly described mutations confirm and further expand the notion that mutations of DNAH11 are a possible cause of PCD in patients with normal axoneme ultrastructure. Thus, genetic evaluation of this region could be used in the diagnostic work-up in difficult cases and to improve the understanding of the basic biology and function of the cilium.

\section{Pifferi*, A. Michelucci" ${ }^{\#}$, M.E. Conidi ${ }^{\#}$, A.M. Cangiotti" P. Simi ${ }^{\#}$, P. Macchia* and A.L. Boner ${ }^{+}$}

*Dept of Paediatrics, and "Cytogenetics and Molecular Genetic Unit, University of Pisa Medical School, Pisa, "Institute of Normal Human Morphology, Electron Microscopy Unit, Umberto I Hospital, University of Ancona, Ancona, and ${ }^{+}$Dept of Paediatrics, University of Verona Medical School, Verona, Italy.

Correspondence: M. Pifferi, Dept of Paediatrics, University of Pisa, Via Roma 67, 56126 Pisa, Italy. E-mail: m.pifferi@med. unipi.it

Support Statement: This work was supported by grants from the Unione Industriale Pisana.

Statement of Interest: None declared. 


\section{REFERENCES}

1 Bush A, Chodhari R, Collins N, et al. Primary ciliary dyskinesia: current state of the art. Arch Dis Child 2007; 92: 1136-1140.

2 Lie H, Ferkol T. Primary ciliary dyskinesia: recent advances in pathogenesis, diagnosis and treatment. Drugs 2007; 67: 1883-1892.

3 Pifferi M, Cangiotti AM, Ragazzo V, et al. Primary ciliary dyskinesia: diagnosis in children with inconclusive ultrastructural evaluation. Pediatr Allergy Immunol 2001; 12: 274-282.

4 Pifferi M, Caramella D, Cangiotti AM, et al. Nasal nitric oxide in atypical primary ciliary dyskinesia. Chest 2007; 131: 870-873.

5 Pifferi M, Montemurro F, Cangiotti AM, et al. Simplified cell culture method for the diagnosis of atypical primary ciliary dyskinesia. Thorax. 2009; 64: 1077-1081.

6 Duriez B, Duquesnoy P, Escudier E, et al. A common variant in combination with a nonsense mutation in a member of the thioredoxin family causes primary ciliary dyskinesia. Proc Natl Acad Sci USA 2007; 104: 3336-3341.

7 Schwabe GC, Hoffmann K, Loges NT, et al. Primary ciliary dyskinesia associated with normal axoneme ultrastructure is caused by DNAH11 mutations. Hum Mutat 2008; 29: 289-298.
8 Piacentini GL, Bodini A, Peroni D, et al. Nasal nitric oxide for early diagnosis of primary ciliary dyskinesia: practical issues in children. Respir Med 2008; 102: 541-547.

9 Bartoloni L, Blouin JL, Pan Y, et al. Mutations in the DNAH11 (axonemal heavy chain dynein type 11) gene cause one form of situs inversus totalis and most likely primary ciliary dyskinesia. Proc Natl Acad Sci USA 2002; 99: 10282-10286.

10 Supp DM, Witte DP, Potter SS, et al. Mutation of an axonemal dynein affects left-right asymmetry in inversus viscerum mice. Nature 1997; 389: 963-966.

11 UniProt Knowledge Database. www.expasy.org/uniprot Date last updated: February 17, 2010.

12 Berkeley Drosophila Genome Project. www.fruitfly.org/seq_tools/ splice.html Date last updated: November 6, 2008.

13 PolyPhen, prediction of functional effect of human nsSNPs. http:// genetics.bwh.harvard.edu/pph Date last updated: February 24, 2010.

14 The Human Gene Mutation Database at the Institute of Medical Genetics in Cardiff. www.hgmd.cf.ac.uk/ac/index.php Date last updated: February 16, 2010.

\section{Exhaled nitric oxide in contrasting population samples of Finnish and Russian Karelia}

\section{To the Editors:}

Considerable differences between countries in prevalence of asthma and atopy have been reported that show association between increased risk and western lifestyle and urbanisation [1]. The allergy substudy of the National FINRISK Study and the Karelia Allergy Study, both carried out in North Karelia, Finland, and the Pitkäranta region, Russia, revealed a significantly higher occurrence of asthma and atopic diseases on the Finnish side, the disparity showing a growing generational trend [2,3]. Despite the geographical neighbourhood, the sensitisation rates for airborne allergens, determined with skin-prick tests or specific immunoglobulin (Ig)E levels, were four-fold in children and two- to three-fold in adults from Finnish Karelia, which may be attributed to the higher level of urbanisation, western type of living conditions and poor microbial environment [4].

Exhaled nitric oxide (eNO) is a noninvasive marker of airway inflammation, and is usually increased in patients with asthma. eNO is strongly associated with atopy in populationbased studies [5-9]. Also, many other factors, such as sex and anthropometric dimensions, tobacco smoking and acute respiratory infections, have been reported to influence eNO levels. To date, there are no reports of the differences in eNO levels between populations with different degrees of urbanisation and living conditions. We postulated that different atopic prevalences between Finnish and Russian Karelia might be reflected in the levels as well as the determinants of eNO. Therefore, in the Karelia Allergy follow-up study in 2007, we measured eNO levels in adult population samples from Finnish and Russian Karelia, and investigated the effect of several demographic, disease-related and environmental factors.
Random samples of adults aged 25-54 yrs were drawn from the population register and electoral rolls in Finnish and Russian Karelia, with participation rates of $61 \%(n=727)$ and $48 \%(n=312)$, respectively. The study included a self-administered questionnaire containing data on demographic, home and living factors and disease history of asthma and atopy, an assay of total and specific IgE against common airborne allergens (birch, mugwort, timothy grass, cat, dog, horse, Cladosporium herbarum and Dermatophagoides pteronyssinus; Phadiatop, UniCAP 1000, Pharmacia Upjohn, Uppsala, Sweden) and measuring eNO by using a portable electrochemical analyser (Niox Mino, Aerocrine AB, Sweden). This device measures eNO during a 10-s exhalation with a constant flow of $50 \mathrm{~mL} \cdot \mathrm{s}^{-1}$, according to the international recommendations. All the measurements were performed during winter, outside the pollen season. Altogether 691 subjects (females $53 \%$ ) in Finland and 305 subjects (females 61\%) in Russia had successful eNO measurements. The mean age was similar in the study samples (40 versus $40 \mathrm{yrs}$, respectively). eNO data were $\log _{10}$ transformed for statistical analysis. The MannWhitney U-test, t-test and linear regression analysis were used for univariate analyses, and general linear methods were used to study the independent effects of the variables in multivariate settings. The study was approved by the coordinating ethics committee of the Helsinki and Uusimaa District Hospital, Finland.

The current overall results concerning higher prevalence of reported asthma, atopic diseases and specific IgE responses in North Karelia, Finland, compared with those observed in the Pitkäranta region, Russia, are described elsewhere ( $T$. Laatikainen, personal communication). The subjects participating in the eNO study in Finnish Karelia showed a higher 